\title{
Evaluating Player Experiences in Location Aware Games
}

\author{
Rod McCall \\ CVAE Department \\ Fraunhofer FIT \\ Sankt Augustin \\ Germany
}

\author{
Anne-Kathrin Braun \\ CVAE Department \\ Fraunhofer FIT \\ Sankt Augustin \\ Germany
}

\author{
Barbara Maria Grüter \\ Center \\ Hochschule Bremen, University of \\ Applied Sciences \\ Bremen
}

Germany rod.mccall@fit.fraunhofer.de anne-

kathrin.braun@fit.fraunhofer.de

\author{
barbara.grueter@hs- \\ bremen.de
}

\begin{abstract}
This workshop will bring together practitioners, students and researchers with an interest in studying player experiences and evaluating playability and usability of location aware games ranging from mixed reality environments through to mobile phone based systems. It will specifically explore the applicability of current HCI practice, how to enhance or develop new techniques and lessons learned from existing studies in the form of methodologies and general design principals.
\end{abstract}

\section{Categories and Subject Descriptors}

H.5.1 [Multimedia Information Systems]: Artificial, augmented, and virtual realities - Audio input/output Evaluation/methodology. H.5.2 [User Interfaces]: Evaluation/methodology - Input devices and strategies Interaction styles - Style guide -, Theory and methods. K.8.0 [General] Games

\section{General Terms}

Quantitative and qualitative methods, Design, Reliability, Human Factors

\section{Keywords}

Augmented Reality (AR), Multimodal Interfaces, Mobile Gaming, Pervasive Gaming, Mixed Reality (MR).

\section{INTRODUCTION}

Location aware technologies such as widespread mobile computers and varying location sensors open up a massive range of possibilities for extending game playing into streets, buildings and even the rural landscape. New and extended forms of location-aware games including mobile or pervasive

\footnotetext{
(c) The Author 2008.
}

Published by the British Computer Society phone games, smart toys, role-playing games as well as Mixed Reality (MR) games all demonstrate promising new forms of game play. Substantial work has also gone into new game concepts, sophisticated technology and viable business models. However, research on the methodological issues of studying mobile player experiences, pervasive game activity and ubiquitous interaction has become necessary. Furthermore, there is also a need to explore the methodological issues in the evaluation of the intertwined, mutually dependent dimensions related to the usability and playability of location-based games. Studying and evaluating player experiences in a mobile setting involves new challenges, as it is often difficult or impossible to apply traditional methods. For example how do we observe the players if they are distributed and always moving? Is it possible to access location-based player experiences? What kind of data do we have to collect for studying mobile player experiences? Is it possible to collect data in an unobtrusive way? And how do we record the actual game processes so that they can be studied later?

This workshop will bring together students, practitioners and researchers with the objective of sharing knowledge, experience and ideas so that the usability issue of location aware games can be more thoroughly addressed.

\section{Background}

Studying player experience in location aware games is often carried out via the analysis of log-files, audio or video recordings, as well as via observations [1] [2]. However, these methods are not satisfactory since they are either rather vague or influence the player experience. Unobtrusive, but yet rich methods need to be found that are able to capture the player's overall experience including the perception of the physical and the virtual part of the game space.

From the perspective of augmented and mixed reality systems (AR/MR), a recent study [3] found that there was little if any regard for usability either within the design or evaluation process, indeed only 14\% of publications from 1998-2004 specifically addressed HCI; while even fewer actually conducted any studies. This is no doubt in part due to the technical challenges posted by such systems, many of which also result in usability problems for example poor tracking, poor object registration or complex devices. 
Several attempts have been made to address the application of HCI principles within MR/AR research and as noted by [3] there are two main approaches, either to adopt the approach of scouring existing AR literature and attempting to extract common guidelines and principals or another approach is to take existing HCI practice and apply them to AR/MR systems. Questions also arise on how to explore the experiences of nongame participants, and how to design and evaluate interaction schemes, that involve a mix of real and virtual objects [4].

In common with tangible interaction, human-computer interaction in location-based games raises the possibility for user experiences, which combine disparate and perhaps previously unconsidered elements and dimensions. The interaction scheme is further complicated by the often diverse range of device, e.g. from mobile phones through to head mounted displays. Furthermore the range of devices also increases the complexity of interaction. This often has the effect of making the users focus on the devices rather than the game-play. These along with many of the themes discussed earlier means that there is now a clear need to place HCI related issues at the heart of location-aware games design and research.

\section{Workshop Challenges and Themes}

Workshop participants will be encouraged to focus on the main themes listed below:

- User interface design issues on multiple device types: from ideas to guidelines and principals

- Devices and modalities, including tangible computing

- Appropriateness of existing HCI work to games interfaces e.g. task analysis, heuristics, interviews and other methods

- Social dimensions of location gaming technologies, from non-player participation to between player communication

- Theoretical issues related space, place and presence

- Contextual issues when designing and evaluating location aware games

- $\quad$ Using participatory design and probes in design and evaluation

- Game design patterns

\subsection{Workshop Structure}

The aim of the workshop is to promote discussion in order to advance knowledge within this growing area. All participants will be asked to submit a 2-4 page position paper and thirty word contributions and benefits statement. Participants will also be encouraged to supply video examples (maximum five minutes) which should contain demonstrations of their technologies and examples of user studies. Authors will also be asked to provide links to up to four key papers. The videos, reference list and position papers will be made available for download around one month prior to the workshop.

All workshop participants will be asked to provide a one slide description of themselves and their work areas, along with a statement of what they intend to present. They will be given two minutes to present this information to the group. This will then be used to identify the main topics during the workshop and the subsequent breakout sessions.
We expect that 8-9 position papers will be accepted for presentation, and those passing a threshold score will be invited to present their work as a poster.

\subsection{Workshop Committee}

Anne-Kathrin Braun is working as a research associate at Fraunhofer FIT in St. Augustin, Germany, in the Collaborative Virtual and Augmented Environments department. Currently she is working as project manager for the TimeWarp Game which is part of IPCity (Interaction and Presence in Urban Environments). Furthermore she has been working on several research projects including CONNECT (Advanced Technologies to connect formal and informal learning environments).

Dr. Barbara Maria Grüter is a psychologist and Professor of Human-Computer Interaction at Hochschule Bremen, University of Applied Sciences. She is a director of research projects on the design and evaluation of mobile technologies and their use since 2001 and on mobile gaming experiences by means of prototyping and play-testing mobile games since 2003 (www.gangs-of-bremen.de). She has been working as a research associate in Psychology and as an assistant professor in Developmental Psychology from 1985 to 2000 at Freie Universität Berlin. During that time she has been a visiting scholar at the University of North Carolina at Chapel Hill, NC, USA from August 1996 to June 1997. She holds a Diplom and a PhD in Psychology (Freie Universität Berlin).

Dr. Rod McCall is a research associate at Fraunhofer FIT. He holds a Ph.D. (Napier), Masters (De Montfort) and Honours Degree (Dundee) in areas related to his research. He is currently the local project manager at FIT for the PEACH (presence research in action) EU coordinated action and has been involved in a variety of EU projects including IPCity, BENOGO, DISCOVER and PERSONA.

\subsection{Post Workshop}

Following the workshop all participants will be invited to submit a full paper or chapter for inclusion in a special issue of a journal or an edited book.

\section{REFERENCES}

[1] Bell, M., Chalmers, M., Barkhuus, L., Hall, M., Sherwood, S., Tennent, P., Brown, B., Rowland, D., and Benford, S. 2006. Interweaving mobile games with everyday life. In Proceedings of the SIGCHI Conference on Human Factors in Computing Systems (Canada, April 2006). CHI '06. ACM, New York.

[2] Fischer, J., Lindt, I., and Stenros, J. Evaluation of Crossmedia Gaming Experiences in Epidemic Menace. 4th International Symposium on Pervasive Gaming Applications (PerGames 2007), June 11 - 12, Salzburg, Austria, 2007.

[3] Dünser, A., Grasset, R., Seichter, H., Billinghurst, M In Proceedings of the 2nd International Workshop on Mixed Reality User Interfaces: Specification, Authoring, Adaptation (MRUI '07), March 11th, Charlotte, NC, USA, 2007.

[4] McCall, R., Herbst, I., Braun, A., and Wetzel, R. The "Where" of Mixed Reality: Some Guidelines for Design. In the Proceedings of the Workshop on Urban Mixed Realities: Technologies, Theories and Frontiers (CHI 2008), Florence, Italy (to appear) 